\title{
Molecular charge distribution and dispersion of electronic states in the contact layer between pentacene and $\mathrm{Cu}(119)$ and beyond
}

\author{
E. Annese, ${ }^{1}$ J. Fujii, ${ }^{1}$ C. Baldacchini, ${ }^{2, *}$ B. Zhou, ${ }^{1}$ C. E. Viol, ${ }^{1}$ I. Vobornik, ${ }^{1}$ M. G. Betti, ${ }^{2}$ and G. Rossi ${ }^{1,3}$ \\ ${ }^{1}$ TASC National Laboratory, INFM-CNR, SS 14, km 163.5, I-34012 Trieste, Italy \\ ${ }^{2}$ Dipartimento di Fisica, Università La Sapienza, P.le A. Moro 2, I-00185 Roma, Italy \\ ${ }^{3}$ Dipartimento di Fisica, Università di Modena e Reggio Emilia, via Campi 213/A, I-41100 Modena, Italy
}

(Received 17 January 2008; revised manuscript received 27 February 2008; published 13 May 2008)

\begin{abstract}
The interaction of pentacene molecules in contact with the $\mathrm{Cu}(119)$ stepped surface has been directly imaged by scanning tunneling microscopy and analyzed by angle resolved photoemission spectroscopy. Interacting molecules, which are in contact with copper, generate dispersive electronic states associated with a perturbed electron charge density distribution of the molecular orbitals. In contrast, the electron charge density of molecules of the pentacene on top of the first layer, which is not in direct contact with the $\mathrm{Cu}$ surface, shows an intramolecular structure very similar to that of the free molecule. Our results indicate that the delocalization of the molecular states in the pentacene/Cu system is confined to the very first molecular layer at the interface.
\end{abstract}

DOI: 10.1103/PhysRevB.77.205417

PACS number(s): 73.20.At, 68.37.Ef, 79.60.Dp

\section{INTRODUCTION}

The understanding of the interaction between planar $\pi$-conjugated organic molecules and metallic surfaces is crucial for the development of future molecular technologies. ${ }^{1-4}$ Recent spectroscopy experiments have provided evidence of the formation of hybrid electron states at the interface between organic molecules and noble metal surfaces. ${ }^{1,4-6}$ Polarization dependent photoemission and density functional theory (DFT) predictions with symmetry-based analysis on model interface systems have identified the hybridization states at the pentacene/Cu interface. ${ }^{5,6}$ Experiments on thick pentacene films (over $100 \AA$ thicknesses) do yield a fully consistent electronic structure with the gas phase spectra, i.e., solid pentacene polycrystalline films are bound by weak electrostatic van der Waals forces. ${ }^{7}$ Issues on the delocalization of the electronic states at the interface and how the interaction affects the molecular charge distribution remain open issues.

Here, we address the transition from the interface to the overlayer pentacene molecular states by a simultaneous analysis of scanning tunneling microscopy (STM) and angle resolved photoelectron spectroscopy (ARPES) with polarized synchrotron radiation results in ultrathin [approximately monolayer $\left.^{8}(\mathrm{ML})\right]$ pentacene films on $\mathrm{Cu}(119)$. Complementary hints on the deformation of molecular charge distribution in the real space and on the description of dispersive electronic states in the reciprocal space clearly show how these effects are strictly confined in the pentacene layer in contact with the metal substrate.

\section{EXPERIMENTAL DETAILS}

In an ultrahigh vacuum condition, we grew pentacene on $\mathrm{Cu}(119)$, which is a vicinal surface of $\mathrm{Cu}(001)$ with $11.45 \AA$ wide terraces separated by monatomic steps. The rationale of this choice of substrate is to obtain an aligned film with pentacene lying flat on the surface and oriented along the $\mathrm{Cu}(119)$ steps. $^{9-12}$ Extended regions of the substrate with uniform steps and terraces were obtained after several cycles of ion sputtering $\left(\mathrm{Ar}^{+}, E=600 \mathrm{eV}\right)$ and annealing at $740 \mathrm{~K}$, which are followed by slow cooling to room temperature (RT). The copper surface quality was checked by both STM and low-energy electron diffraction. ${ }^{9}$ Pentacene was deposited in situ by evaporating pentacene from a resistively heated quartz crucible at a rate of $\sim 0.1 \mathrm{~nm} / \mathrm{min}$ in a residual pressure always $<5 \times 10^{-10}$ mbar. During the deposition, the substrate was kept either at $370 \mathrm{~K}$ to limit the adsorption to a single layer ${ }^{8}$ or at RT for a higher coverage. ARPES spectra were measured at RT at the APE-INFM low-energy beamline at Elettra by using a SES2002 electron energy analyzer operated at $15 \mathrm{meV}$ energy resolution and $0.2^{\circ}$ angular resolution. The photon energy was $18 \mathrm{eV}$. STM measurements were acquired in constant current mode at RT with a tungsten tip, which is capable of atomic resolution. ${ }^{13}$ These growth procedures resulted in pentacene arrays lying flat on the substrate, with the long molecular axis parallel to the step edges. ${ }^{11}$

\section{PHOTOEMISSION RESULTS}

Photoelectrons are excited by photons with the polarization vector oriented either perpendicular [Figs. 1(a) and 1(b)] or parallel [Fig. 1(c)] to the long molecular axis of the pentacene molecules, which are uniformly aligned along the $\mathrm{Cu}(119)$ steps (experimental geometry reported in Ref. 5). Representative photoemission results for different pentacene coverages $^{8}$ on $\mathrm{Cu}(119)$ are collected in Fig. 1(a) for a single layer at saturation coverage $(1 \mathrm{ML})$ and for $\sim 2 \mathrm{ML}$, which are compared to the spectrum of the clean $\mathrm{Cu}(119)$ surface. The spectra are integrated over $10^{\circ}$ around the $\bar{\Gamma}$ point. The spectral enhancement, $I_{1}$, close to the Fermi level $\left(E_{F}\right)$ is detected at the completion of the saturated layer and it is due to the partial filling of the lowest unoccupied molecular orbitals (LUMOs) hybridized with the substrate electronic states as observed in pentacene, ${ }^{5,6}$ in 3,4,9,10-perylenetetracarboxylic acid dianhydride (PTCDA), ${ }^{4}$ and in fullerene ${ }^{14}$ single layers deposited on metal substrates. A second weak interface peak, $I_{3}$, appears at $\sim 1.6 \mathrm{eV}$ : it has been 

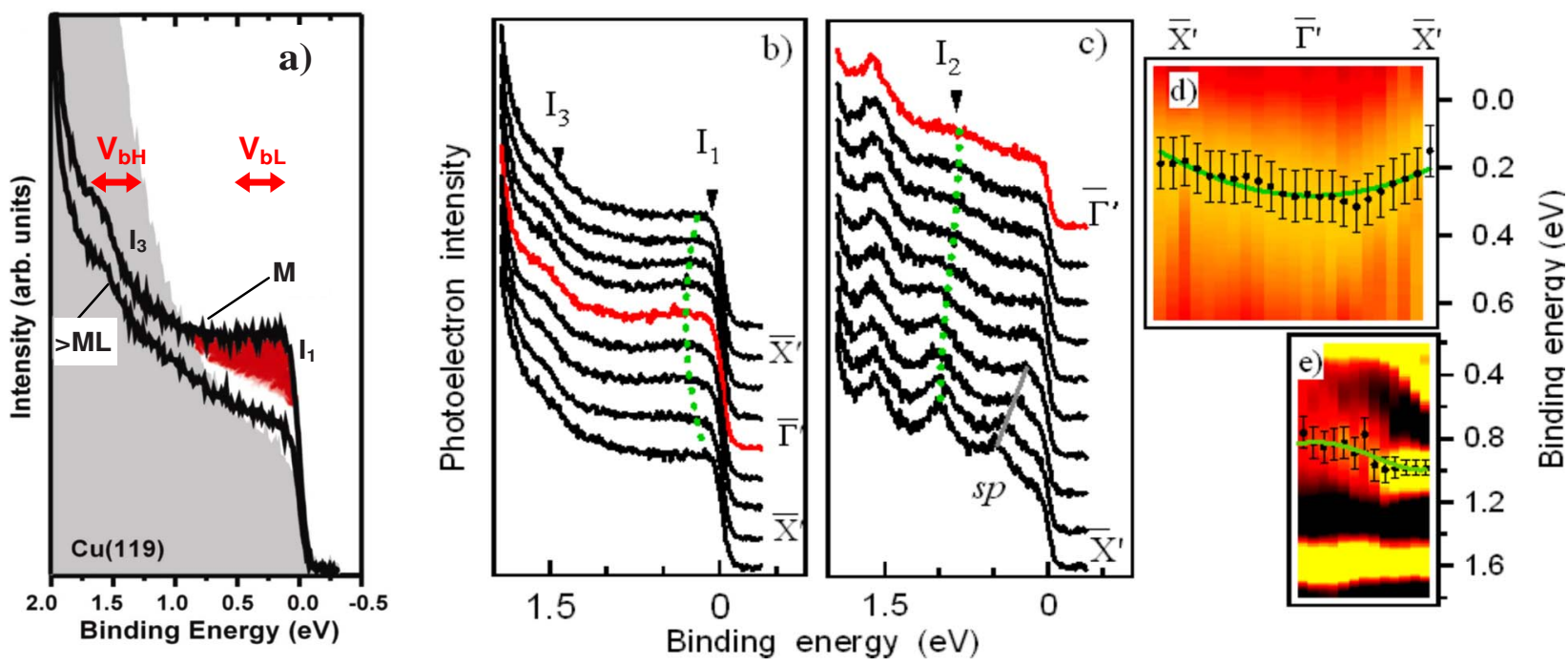

FIG. 1. (Color online) ARPES spectra at $h \nu=18 \mathrm{eV}$ with the light polarization [(a) and (b)] perpendicular and (c) parallel to the long molecular axis; (a) angle integrated spectra around $\bar{\Gamma}$ for pentacene on $\mathrm{Cu}(119)$ ): clean substrate (gray shaded), (ML) (Ref. 8) and higher coverage; $\sim 2$ ML: (b) $I_{1}$ (close to $E_{F}$ ) and HOMO-derived spectral features, $I_{3}$, along the $\bar{\Gamma}^{\prime}-\bar{X}^{\prime}$ direction around $\bar{\Gamma}$. The green dashed line is the parabolic fitting of the $I_{1}$ peak position; (c) extra molecular interface state, $I_{2}$ (Ref. 5), and the residual substrate $s p$ band (gray solid line) (Ref. 16) dispersing along $\bar{\Gamma}^{\prime}-\bar{X}^{\prime}$. The green dashed line is the fitting curve of the $I_{2}$ peak position; (d) spectral intensity of the $I_{1}$ molecular interface state along the $\bar{\Gamma}^{\prime}-\bar{X}^{\prime}$ direction after subtracting the spectrum beyond the $\bar{X}^{\prime}$ point and normalizing to the $I_{1}$ peak. The green solid line is the result of the fitting of the $I_{1}$ peak position (filled circle); (e) the second derivative plot of the spectral intensity of $I_{2}$. The green solid line is the fitting curve of the peak energy position of the second derivative of the spectral intensity (filled circle).

attributed to an interface pentacene state derived from the highest occupied molecular orbital (HOMO) state. ${ }^{5}$

Panels (b) and (c) of Fig. 1 display the ARPES spectra of a highly ordered pentacene layer with a $3 \times 7$ superstructure. ${ }^{9-11,15}$ The Surface Brillouin Zone (SBZ) along $\bar{\Gamma}-\bar{X}^{\prime}$ (marked as $\bar{\Gamma}^{\prime}-\bar{X}^{\prime}$, parallel to the substrate steps) is seven times smaller than the $\mathrm{Cu}(100) \mathrm{SBZ}$. The spectral features in Fig. 1(b) are observed in the first reduced SBZ in the $\bar{\Gamma}^{\prime}-\bar{X}^{\prime}$ direction, while the spectra in Fig. 1(c) are taken in the fourth reduced SBZ $\bar{\Gamma}^{\prime}-\bar{X}^{\prime}$, which, in fact, overlaps the substrate $\bar{X}$ point. In Fig. 1(c), a further interfacial peak, $I_{2}$, is observed at $\sim 1 \mathrm{eV}$ binding energy and previously assigned to a LUMO-derived state. ${ }^{5,6}$

A feeble intensity modulation in plane along $\bar{\Gamma}^{\prime}-\bar{X}^{\prime}$ [see Fig. 1(b) and 1(c)] is observed for the pentacene induced structures, $I_{n}$. Following peak $I_{1}$, the maximum intensity is observed at normal emission and it disappears beyond the first $\bar{X}^{\prime}$ points. The evaluation of the $I_{1}$ peak energy position is difficult due to the residual substrate intensity, ${ }^{16}$ which shows a $k$-dependent intensity modulation in this energy region $(0-1.5 \mathrm{eV})$. In order to evaluate the energy position along $\bar{\Gamma}^{\prime}-\bar{X}^{\prime}$ of the $I_{1}$ peak, the spectral intensity is deduced from the spectra by subtracting at each spectrum one beyond the first $\bar{X}^{\prime}$ point. The spectra normalized by the peak intensity and the estimated peak position (dots) are displayed in Fig. 1(d). A parabolic curve is used for the fitting curve (solid line). The curve is not centered at $\bar{\Gamma}^{\prime}$ due to the angular dependence of the substrate background. The peak $I_{2}$ disperses from $\bar{X}^{\prime}$ to $\bar{\Gamma}^{\prime}$ [see Fig. 1(c)] in the vicinity of $\bar{X}$, i.e., overlapping the dispersion of the pro- jection of the substrate $s p$ bulk band moving toward the $\bar{\Gamma}^{\prime}$ point, ${ }^{16}$ as indicated by the solid line in Fig. 1(c). The $I_{2}$ peak position (dots) is determined by using the second derivative of the spectra [Fig. 1(e)]. A polynomial curve is used for the fitting of the $I_{2}$ peak energy position and the results (solid line) are shown in the same panel. The $I_{3}$ (HOMO-derived) feature at $\sim 1.6 \mathrm{eV}$ binding energy (very weak intensity at normal emission [Figs. 1(a) and 1(b)], but enhanced off normal [Fig. 1(c)]), does not exhibit dispersion within our accuracy.

The energy dispersion of the $I_{1,2}$ peaks in the direction parallel to the short molecular axis (e.g., perpendicular to the steps of the substrate) could not be detected within our accuracy, which is consistent with the one-dimensional character of the state pertinent to the pentacene chains. The tiny energy dispersion along $\bar{\Gamma}^{\prime}-\bar{X}^{\prime}$ can be ascribed to the charge delocalization along the pentacene chains, which is mediated by the metallic substrate at the interface. As the molecular layers pile up, at higher pentacene coverages, $I_{1}$ and $I_{2}$ are not detected, while the HOMO-derived peak $I_{3}$ increases in intensity. ${ }^{7,9}$

\section{SCANNING TUNNELING MICROSCOPY RESULTS}

The STM images of Figs. 2(a) and 2(b) were acquired with atomic resolution at the relevant values of the bias voltages $\left(\left|V_{b L}\right|,\left|V_{b H}\right|\right)$, which correspond to the binding energies of the molecular interface states $I_{1}$ and $I_{3}$ in the photoemission spectra [Fig. 1(a)]. Bias voltages $V_{b}$ are referred to the sample, i.e., negative bias.

Isolated pentacene molecules $\left(0.05\right.$ molecule $\left./ \mathrm{nm}^{2}\right)$ are imaged in Figs. 2(a) and 2(b). The $z$ scale is multiplied by 4 


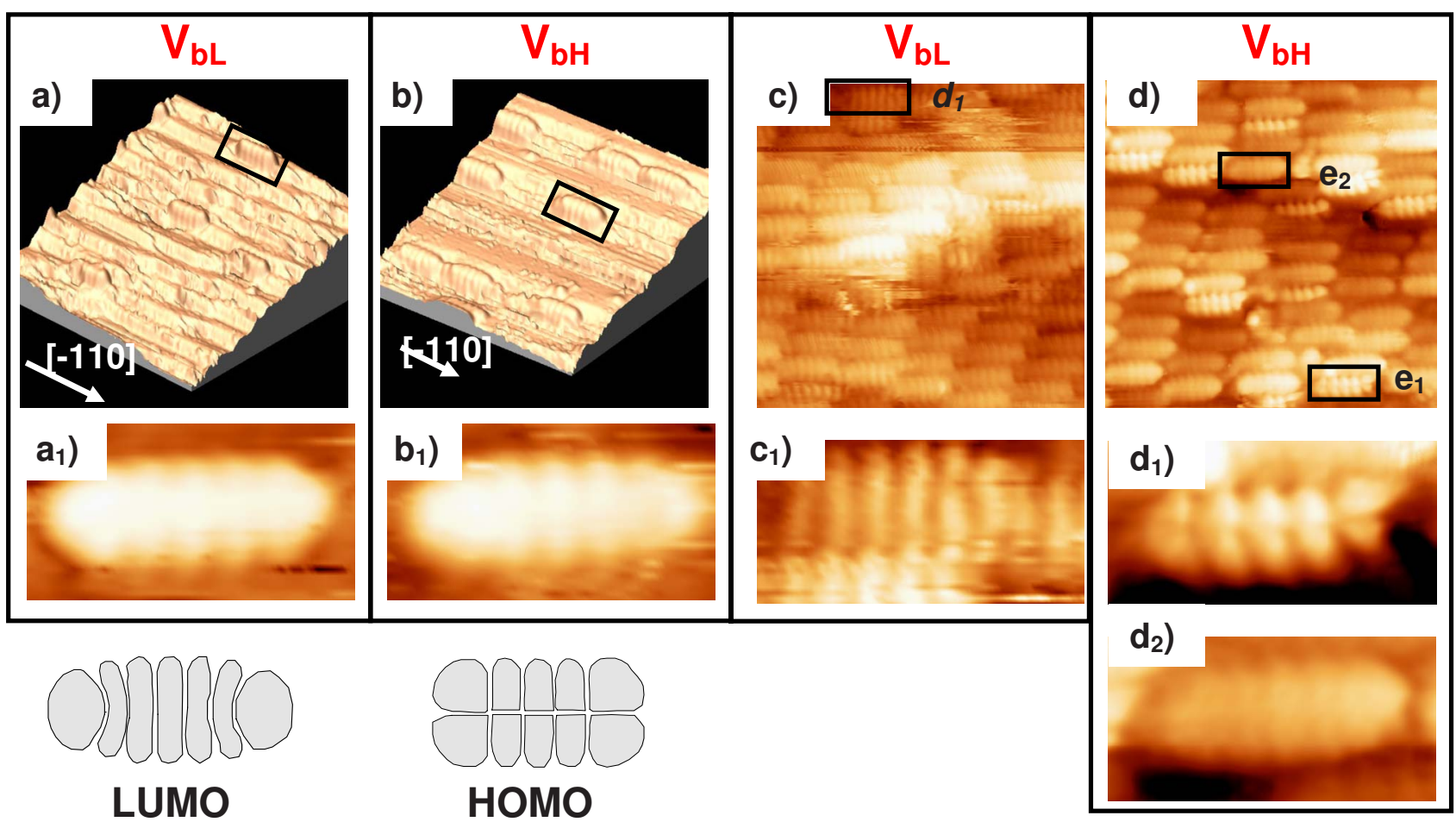

FIG. 2. (Color online) Bias-dependent STM images of pentacene molecules on $\mathrm{Cu}(119)$ at $[(\mathrm{a})$ and (b)] submonolayer coverage and [(c) and (d)] 1.1 ML coverage; size: $9.1 \times 10.0 \mathrm{~nm}^{2}$; in (a) and (c), the bias corresponds to LUMO-derived features $\left(V_{b L}\right)$, in $(b)$ and $(d)$, the bias corresponds to HOMO-derived features $\left(V_{b H}\right)$, as marked in the ARPES spectra; $\left(\mathrm{a}_{1}\right),\left(\mathrm{b}_{1}\right),\left(\mathrm{c}_{1}\right)$ and $\left(\mathrm{d}_{1-2}\right)$ are zoom-in to the selected molecules (rectangles) in images (a)-(d); the insets are the HOMO and LUMO calculated electronic density distributions. Bias and tunneling current of the images: (a) $V_{b L}=-0.054 \mathrm{~V}, I=0.67 \mathrm{nA}$ (b), $V_{b H}=-1.4 \mathrm{~V}, I=3.0 \mathrm{nA}$, (c), $V_{b L}=-0.24 \mathrm{~V}, I=23 \mathrm{nA}$, and (d) $V_{b H}=-1.3 \mathrm{~V}, I$ $=0.67 \mathrm{nA}$.

with respect to the $x$ and $y$ scales to magnify the step structure. The size of the individual adsorbed pentacene molecules is $1.62( \pm 0.08)$ and $0.70( \pm 0.08) \mathrm{nm}$ for the long and short molecular axes, respectively. The molecular diffusion along the terraces at RT was monitored by measuring the displacement of a molecule between subsequent STM images. We estimate an upper limit of the molecular diffusion speed of $0.025 \mathrm{~nm} / \mathrm{s}$, which is sufficiently low to guarantee the high image resolution.

The shape or the size of the tip apex is known to affect the aspect of the images. We could establish tip conditions (the one exploited for the shown images), which was stable and provided the same image quality when the bias was changed from 0 to $-1.8 \mathrm{~V}$ at a fixed tunneling current. This tip condition was metallic. It is well established that STM images with a metallic tip correspond to the local charge density, i.e., the electron states of organic molecules on metallic substrate. ${ }^{17-19}$

The data in Figs. 2(c) and 2(d) are relative to a molecular density of $\sim 0.80$ molecule $/ \mathrm{nm}^{2}$, i.e., a coverage exceeding the monolayer $(\sim 1.1 \mathrm{ML})$. The substrate step structure (average terrace width of $11.45 \AA$ ) cannot be observed anymore. The molecules form flat terraces whose average width is much wider than that of the substrate terraces. The periodicity in the image is determined by the size of the molecules and not by the substrate step size. The only memory of the substrate step structure is the molecular orientation. At the edge of the terraces, the second layer molecules are ob- served. Since there are no molecules perpendicular to the steps, the structure is not driven by the formation of substrate facets, which have been previously shown for annealed multilayer pentacene films on the same system. ${ }^{20}$

The isolated pentacene molecules on copper, as imaged with both $V_{b L}$ and $V_{b H}$, show a defined molecular charge density even for a bias voltage below the energy of the $I_{3}$ interface states, confirming the presence of the interacting states observed in the photoemission experiment. The pentacene molecules in Figs. 2(a) and 2(b) exhibit seven charge protrusions per molecule separated by $0.23( \pm 0.02) \mathrm{nm}$ along the main molecular axis and a nodal plane along the long molecular axis is absent. When compared to the insets, which reproduce the scheme of the HOMO and LUMO electronic densities for a free pentacene molecule, ${ }^{18}$ one can conclude that both high and low bias images show a molecular charge with a different distribution compared to the HOMO molecular charge in the free molecule.

The seven protrusions charge pattern could also be observed at a higher pentacene density when imaging with low bias $V_{b L}$ [Fig. 2(c)]. When imaging at high bias $V_{b H}$, two different types of molecular charge pattern can be distinguished: one corresponds to the topmost molecules, which are not surrounded by other molecules besides the ones underneath [Fig. $\left.2\left(\mathrm{~d}_{1}\right)\right]$, as recognized by the local topography; the second corresponds to molecules that are aligned within a dense pentacene layer in direct contact with the substrate [Fig. $\left.2\left(\mathrm{~d}_{2}\right)\right]$. The $V_{b H}$ image [Fig. $\left.2\left(\mathrm{~d}_{1}\right)\right]$ of the topmost mol- 

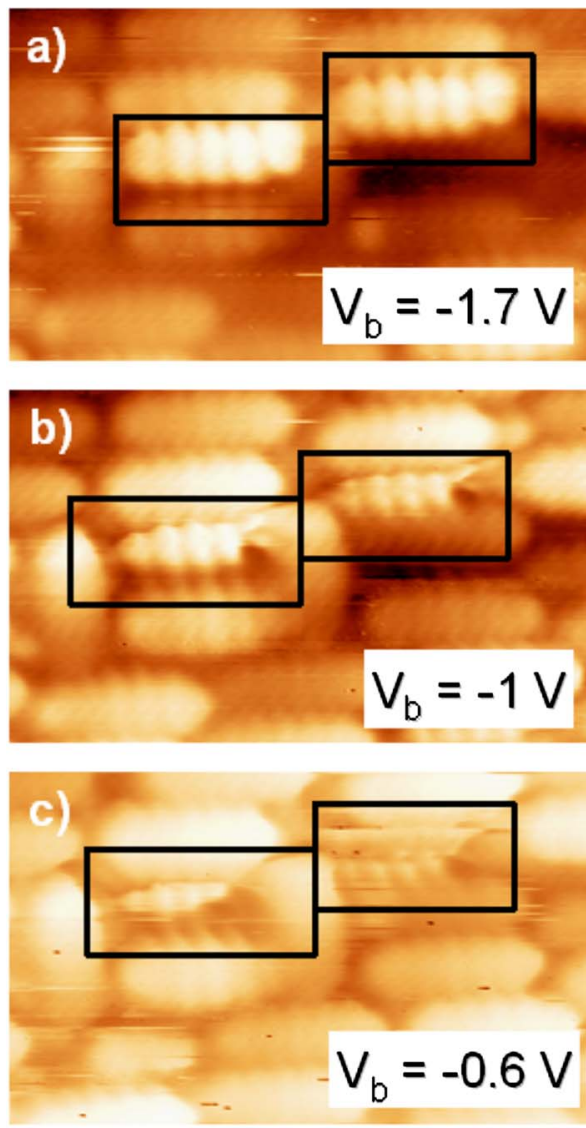

FIG. 3. (Color online) Bias-dependent STM images (size: 5.4 $\times 3.7 \mathrm{~nm}^{2}$ ) for $1.1 \mathrm{ML}$ film: as the bias is reduced [(a)-(c)] the topmost molecule (rectangle) apparently disappears.

ecules shows five protrusions separated by $0.31( \pm 0.07) \mathrm{nm}$ and a clearly distinguishable nodal plane along the long molecular axis, mimicking the HOMO features of the free molecule. The molecules belonging to the interface layer [Fig. $2\left(\mathrm{~d}_{2}\right)$ ] also show a charge density distribution similar to the case of the molecules in Fig. 2(b) that therefore represents mostly the interaction states with the substrate.

In Figs. 3(a)-3(c), the bias-dependent images of a 1.1 ML film are reported [the same as in Fig. 2(d)]. As the bias is reduced in absolute value from $1.7 \mathrm{~V}$ [Fig. 3(a)] to $0.6 \mathrm{~V}$ [Fig. 3(c)], the image of the topmost molecule progressively shrinks and disappears.

\section{DISCUSSION}

The dispersion of interface electronic states and the perturbed molecular charge distribution are both signatures of the pentacene-Cu hybridization at the interface. The photoemission intensity close to $E_{F}$ (Refs. 5 and 6 ) and the dispersion of these LUMO-derived states along $\bar{\Gamma}^{\prime}-\bar{X}^{\prime}$ confirm and extend previous results for $\pi$-conjugated molecules interacting with metal substrates. ${ }^{1,4-6}$ The complementary STM data on the changes of the molecular electron density due to the interaction with the substrate give evidence of specific distortion of the molecular states at the interface that cannot be mapped onto free-molecule-like orbitals. ${ }^{1,17,21,22}$ The seven- lobe charge distribution is measured at the $I_{3}$ and $I_{1}$ binding energies. The observed charge redistribution at the $I_{3}$ interface state, i.e., HOMO-derived state, may be induced by the presence of the energetically adjacent interface electronic states $I_{1}$ and $I_{2}$, as also suggested by DFT calculations. ${ }^{6}$ On the other hand, a similar appearance of molecular orbital internal structures at both high and low biases may indicate that molecular states are involved in the bonding to the substrate, with a similar hybridization scheme as recently reported in pentacene interacting with a single gold atom. ${ }^{17}$

ARPES data in the adopted geometry exhibit an odd symmetry ${ }^{5}$ of the $1.6 \mathrm{eV}$ molecular states with respect to a plane $\sigma$, which contains the long molecular axis and the normal to the substrate. The seven-lobe molecular charge density for the pentacene molecules in contact with the metal surface displayed by STM both for $V_{b H}$ and $V_{b L}$ does not display the same symmetry with respect to the same plane $\sigma$. However, the STM image shows the local electronic density and is not sensitive to the phase of the molecular states. A modification of the system parity may be induced by the intermolecular interaction since a 1.1 ML pentacene sample presents a two-dimensional arrangement with molecules close to each other. According to these considerations, the comparison between the parities revealed by the two techniques is not straightforward.

Identifying molecules beyond the interface allows us to discuss the changes of their electronic states as a function of molecular layers. Molecules lying above the interface layer are easily singled out in the STM topography. Their electronic structure is derived by the bias dependence of the STM images of specific molecules. At low absolute values of the bias, only molecules hybridizing with the substrate (binding energy close to $E_{F}$ ) can provide tunneling current. By imaging the $\sim 1.1 \mathrm{ML}$ film with the absolute bias decreasing from 1.7 to $0.6 \mathrm{~V}$ [Figs. 3(a)-3(c)], one observes, in fact, the disappearance of the topmost pentacene molecule charge density and at the same time the appearance of the molecules underneath. The molecules in contact with the substrate remain clearly visible at all bias values, always displaying the same electronic charge distribution. The vanishing charge density of the top molecules occurs, in fact, for the values of the bias in the hole-injection barrier $(1.5 \mathrm{eV})$, indicating that the interaction between these molecules and the substrate is weak or mediated by the interface layer.

Previous results on pentacene thick film growth have shown a transition from flat to tilted geometry, where low density brick or wave phases are observed and predicted in the case of pentacene grown on different copper surfaces. ${ }^{23,24}$ Previous STM results at the surface of a thin pentacene film (>2 ML) on $\mathrm{Cu}(119)$ (Ref. 25) show a flat pentacene geometry. The present findings confirm that the isolated pentacene molecules of the second layer are flat on the surface [aligned along the underlying molecular interface layer, i.e., along the $\mathrm{Cu}(119)$ step edges] and with a molecular electron distribution that looks freelike molecules. The upper face of the contact pentacene layer may present a modified charge density, with the seven-lobe distribution, which minimizes the face-to-face repulsion between two adjacent pentacene molecules favoring the flat geometry of the second layer instead of the tilting of the molecules, as observed in pentacene thick 
layers. The maximum expected tilt angle along the short molecular axis, which is estimated from STM images, is less than $10^{\circ}$, which is much smaller than what is observed in other pentacene/copper systems. ${ }^{24}$ The strength of the interaction between molecules in the overlayer and at the interface is enough to drive the adsorption geometry but not to perturb the electronic distribution of the topmost molecules, which are not in direct contact with the metal substrate. Similar molecular electronic distributions have been reported for pentacene and $\mathrm{Cu}(100)$ when decoupled by an insulating $\mathrm{NaCl}$ intralayer film from the metallic substrate. ${ }^{18}$

\section{CONCLUSIONS}

We have identified a peculiar charge density distribution of the pentacene molecules in contact with the $\mathrm{Cu}(119)$ vici- nal surface. The perturbed molecular orbitals, i.e., the partially populated dispersive LUMO-derived states and the HOMO-derived states, is strictly limited to the contact layer. The second layer molecules are weakly bound to the contact layer but preserve the in-plane orientation and the alignment along the substrate template. Their charge density distribution mimics free pentacene molecular orbitals. In the reciprocal space, the molecular charge distortion gives rise to the electronic states localized at the interface, with a tiny dispersion along the pentacene long molecular axis.

\section{ACKNOWLEDGMENTS}

Funding was provided by MIUR through PRIN2004 and by the University of Rome La Sapienza.
*Present address: Biophysics \& NanoScience Centre and CNISM, Università della Tuscia, Largo dell'Università, 01100 Viterbo, Italy.

${ }^{1}$ R. Temirov, S. Soubatch, A. Luican, and F. S. Tautz, Nature (London) 444, 350 (2006).

${ }^{2}$ H. Kakuta, T. Hirahara, I. Matsuda, T. Nagao, S. Hasegawa, N. Ueno, and K. Sakamoto, Phys. Rev. Lett. 98, 247601 (2007).

${ }^{3}$ M. Eremtchenko, J. A. Schaefer, and F. S. Tautz, Nature (London) 425, 602 (2003).

${ }^{4}$ Y. Zou, L. Kilian, A. Shoell, Th. Schmidt, R. Fink, and E. Umbach, Surf. Sci. 600, 1240 (2006).

${ }^{5}$ C. Baldacchini, C. Mariani, M. G. Betti, I. Vobornik, J. Fujii, E. Annese, G. Rossi, A. Ferretti, A. Calzolari, R. Di Felice, A. Ruini, and E. Molinari, Phys. Rev. B 76, 245430 (2007).

${ }^{6}$ A. Ferretti, C. Baldacchini, A. Calzolari, R. Di Felice, A. Ruini, E. Molinari, and M. G. Betti, Phys. Rev. Lett. 99, 046802 (2007).

${ }^{7}$ C. Baldacchini, C. Mariani, M. G. Betti, L. Gavioli, M. Fanetti, and M. Sancrotti, Appl. Phys. Lett. 89, 152119 (2006).

${ }^{8} \mathrm{We}$ define as the monolayer (1 ML) a saturation coverage of pentacene obtained by keeping the substrate at $370 \mathrm{~K}$; it corresponds to a closed packed molecular structure with a long-range $3 \times 7$ ordering (Refs. 9-11) the molecular density at this coverage is $\sim 0.7$ molecule $/ \mathrm{nm}^{2}$; a higher coverage is obtained by additional evaporation to the substrate kept at room temperature, the nominal thickness is estimated from evaporation time $(\geqslant 2$ $\mathrm{ML})$; submonolayer coverage $(<1 \mathrm{ML})$ corresponds to a molecular density of 0.05 molecule $/ \mathrm{nm}^{2}$.

${ }^{9}$ C. Baldacchini, C. Mariani, and M. G. Betti, J. Chem. Phys. 124, 154702 (2006).

${ }^{10}$ L. Gavioli, M. Fanetti, M. Sancrotti, and M. G. Betti, Phys. Rev. B 72, 035458 (2005).
${ }^{11}$ L. Gavioli, M. Fanetti, D. Pasca, M. Padovani, M. Sancrotti, and M. G. Betti, Surf. Sci. 566-568, 624 (2004).

${ }^{12}$ M. Fanetti, L. Gavioli, M. Sancrotti, and M. G. Betti, Surf. Sci. 252, 5568 (2006).

${ }^{13}$ G. Panaccione, J. Fujii, I. Vobornik, G. Trimarchi, N. Binggeli, A. Goldoni, R. Larciprete, and G. Rossi, Phys. Rev. B 73, 035431 (2006).

${ }^{14}$ A. Goldoni, C. Cepek, E. Magnano, A. D. Laine, S. Vandrè, and M. Sancrotti, Phys. Rev. B 58, 2228 (1998).

${ }^{15}$ C. Baldacchini, V. Corradini, M. G. Betti, and C. Mariani, Surf. Sci. 566-568, 613 (2004).

${ }^{16}$ C. Baldacchini, L. Chiodo, F. Allegretti, C. Mariani, M. G. Betti, P. Monachesi, and R. Del Sole, Phys. Rev. B 68, 195109 (2003).

${ }^{17}$ J. Repp, G. Meyer, S. Paavilainen, F. Olsson, M. Persson, Science 312, 1196 (2006).

${ }^{18}$ J. Repp, G. Meyer, S. M. Stojkovic, A. Gourdon, and C. Joachim, Phys. Rev. Lett. 94, 026803 (2005).

${ }^{19}$ Z. T. Deng, H. Lin, W. Ji, L. Gao, X. Lin, Z. H. Cheng, X. B. He, J. L. Lu, D. X. Shi, W. A. Hofer, and H.-J. Gao, Phys. Rev. Lett. 96, 156102 (2006).

${ }^{20}$ M. Fanetti, L. Gavioli, and M. Sancrotti, Adv. Mater. (Weinheim, Ger.) 18, 2863 (2006).

${ }^{21}$ V. M. Hallmark, S. Chiang, K.-P. Meinhardt, and K. Hafner, Phys. Rev. Lett. 70, 3740 (1993).

${ }^{22}$ J.-W. Wang, K.-H. Wu, W.-S. Yang, X.-J. Wang, J. T. Sadowski, Y. Fujikawa, and T. Sakurai, Surf. Sci. 579, 80 (2005).

${ }^{23}$ M. Satta, S. Iacobucci, and R. Larciprete, Phys. Rev. B 75, 155401 (2007).

${ }^{24}$ S. Söhnchen, S. Lukas, and G. Witte, J. Chem. Phys. 121, 525 (2004).

${ }^{25}$ E. Annese, C. E. Viol, B. Zhou, J. Fujii, I. Vobornik, C. Baldacchini, M. G. Betti, and G. Rossi, Surf. Sci. 601, 4242 (2007). 